\title{
A CASE REPORT OF EXTRACORPOREAL MEMBRANE OXYGENATION AND HEMADSORBER THERAPY IN SEPTIC PATIENT WITH MULTIORGAN INJURY
}

\author{
Anna Klesmite* Roberts Leibuss*,**, Baiba Arklina*,**, Larisa Semcenko**, Martins Kalejs*,*** Eva Strike*,** \\ Peteris Stradins*,*** \\ * Riga Stradins University, Latvia, Dzirciema street 16, Riga, Latvia \\ ** Pauls Stradins Clinical University Hospital, Latvian Centre of Cardiology, 39th Department of Cardiac surgery, \\ anaesthesiology and intensive care unit; Pilsonu street 13, Riga, Latvia \\ *** Pauls Stradins Clinical University Hospital, Centre of Cardiac Surgery; Pilsonu street 13, Riga, Latvia
}

\begin{abstract}
Summary
Diagnostic microbiology and management of sepsis have advanced, even though the complication of multiorgan dysfunction remains a significant cause of morbidity and mortality. An estimated incidence of sepsis in 2017 is about 48 million a year, including 11 million sepsis- related deaths, which represents $19.7 \%$ of all global deaths. $(1,2)$ Acute respiratory distress syndrome (ARDS) is a devastating complication of sepsis, conventionally, a substantial number of patients require mechanical ventilation (MV) to avert hypoxemia and hypercapnia. However, MV per se can cause lung injury, accelerating the disease progression. The use of extracorporeal membrane oxygenation (ECMO) in the management of ARDS has grown considerably in the past decade. More recently, an increasing popularity of spontaneous breathing and awake patients undergoing ECMO is seen, as well as the use of various types of hemadsorbers for reducing pathologically increased inflammatory response.(3) The purpose of this case report is to review airway and multiorgan support management during ECMO.
\end{abstract}

Keywords: veno-venous extracorporeal membrane oxygenation, "awake" ECMO, ARDS, multiple organ dysfunction syndrome

\section{AIM OF DEMONSTRATION}

The aim of this article is to illustrate a case with extracorporeal cytokine adsorber combined in extracorporeal membrane oxygenation due to acute respiratory distress syndrome followed by influenza A type superinfection. Spontaneously breathing patient with a primary cardiac pathology received both pharmacological and mechanical multiorgan support therapy.

\section{CASE REPORT}

A case report of a 38-year old male patient, $67 \mathrm{~kg}$, $174 \mathrm{~cm}$. Initially admitted to Pauls Stradins Clinical University Hospital, Riga, Latvia (table nr.1) during an influenza epidemic. The patient was complaining of chest pain, shortness of breath, febrile temperature for a month. Physical examination showed respiratory rate (RR) 20 times per minute, hemodynamically stable, Glasgow Coma Scale (GCS) 15 points, febrile temperature. First evaluation of quick Sequential Organ Failure Assessment (qSOFA) was 0 points, that represents a risk of bad outcome $<1 \%$. This score is used to identify patients with suspected infection who are likely to develop sepsis or septic shock. Preliminary diagnosis: infective endocarditis. Subsequently the patient was hospitalized in the Cardiology unit for 10 days, where further diagnostics and treatment were made.
A blood culture detected Streptococcus oralis sepsis. On $3^{\text {rd }}$ admission day repeated echocardiogram (picture nr.1) showed aortic valve leaflet sclerosis with additional formation on non-coronary cusp $1,1 \times 1,5 \mathrm{~cm}$, aortic valve regurgitation IV, mitral valve regurgitation III, ejection fraction of $60 \%$, ascending aorta aneurysm, tricuspid valve regurgitation II, TAPSE $22 \mathrm{~mm}$, medium pulmonary hypertension. The patient deteriorated on day 5 presenting with respiratory tract superinfection symptoms. After two days a positive ribonucleic acid test of type A influenza virus was approved and a diagnosis of ARDS was made. Symptoms progressed with third-degree atrioventricular block and generalized seizure; no persistent neurological deficits remained. The patient was transferred to Cardiac Intensive Care Unit (ICU) after showing signs of pulmonary edema, tachypnea, hemodynamically compromised, oxygen saturation $78-80 \%$, febrile temperature. Laboratory results showed decompensated respiratory acidosis (table nr.2.) and computer tomography a typical ARDS finding (picture nr.2). A repeated qSOFA evaluation showed an increased risk of a bad outcome, $<13 \%$. For stratifying severity of acute lung injury and patient selection for ECMO, Murray scored 3.25 points with high lethality risk, $>80 \%$.

The next day the patient was transferred to Cardiothoracic surgery ICU, where a veno-venous (v-v) ECMO (Bio-Console 560, Medtronic, USA) 
was implanted. It was used for six days providing an opportunity for lung recovery to increase better postoperative outcome before heart surgery. During the first two days $\mathrm{v}-\mathrm{v}$ ECMO therapy was combined with CytoSorb adsorber (CytoSorbents Europe GmbH, USA) to reduce the cytokine levels from sepsis. After $v-v$ ECMO implantation blood gas analysis and general clinical condition improved, it was possible to maintain spontaneous ventilation with a high flow oxygen mask, continuing basic treatment and rehabilitation. Following improved lung parameters, planned explanation took place on hospitalization day 16. Risk scores at the time showed- ASA IV, CV Euroscore $4.4 \%$, APACHE II 12\% (calculated at the beginning of the ICU admission to help determine the patient's mortality risk). Patient proceeded to a sternotomy on hospitalization day 24 with mitral and aortic bioprosthetic valve replacement. The patient was discharged from the Cardiothoracic surgery ICU in 9 days with the total length of hospital stay of 40 days. Definitive diagnosis: infective endocarditis with vegetations on aortic and mitral valves; severe mitral and aortic valve insufficiency; Streptococcus oralis sepsis, influenza A virus pneumonia, ARDS, transitory third-degree atrioventricular block, ascending aorta aneurysm. In six months, the patient was readmitted for successful elective ascending aorta replacement.

\section{DISCUSSION}

The case report illustrated a combined therapy with cytokine adsorber in extracorporeal membrane oxygenation on spontaneous breathing patient due to acute respiratory distress syndrome followed by influenza A type superinfection. The case was even more complex due to acute infectious endocarditis affecting both the aortic and mitral valves and causing severe regurgitation of both valves which furthermore worsened patients lung condition due to congestion in the lungs. In literature, no consensus for a polymorbid patients is made for the best strategy for airway management during ECMO. As the current case showed, it is possible to maintain spontaneous breathing during combined therapy with extracorporeal membrane oxygenation in ARDS patients with infective endocarditis.

Since influenza A (H1Nl) pandemic in 2009, it has been widely used for potentially reversible acute respiratory failure. In Latvia, the first $v-v$ ECMO was used for ARDS treatment in 2008.

Extracorporeal Life Support organization recommends early tracheostomy and low-pressure settings for patients with influenza caused pneumonia during $\mathrm{v}-\mathrm{v}$ ECMO.(4) Based on literature from 13 studies with 494 patient's median duration of ECMO was 10 days (in our case six), mechanical ventilation 19 days (in our - noninvasive ventilation (NIV) one day), length of stay in ICU 33 days (in our - 22 days).(5) Treatment remains expensive, requires prolonged ventilation support and intensive therapy, with high associated mortality compared to spontaneous ventilation.
That reduces the need for sedation, risk of cognitive decline, length of stay in ICU and possibility of earlier rehabilitation, as well as the patient continues peroral nutrition, supporting physiologic needs.(6) In our case, the heart-lung complicated patient received step-down approach in airway management (when NIV was ineffective, ECMO was used). During the running of ECMO, the protective ventilation strategy can be employed without worrying about hypoxemia and carbon dioxide retention. There is a large body of evidence showing that protective ventilation with low tidal volume, high positive end-expiratory pressure, nitric oxide inhalation and prone positioning can provide benefits on mortality outcome. More recently, there is a trend on the use of awake and spontaneous breathing for patients undergoing ECMO, which is thought to be beneficial in terms of early rehabilitation in ICU. Hemoadsorption with Cytosorb during ECMO therapy can be used to decrease patient's cytokine levels in case of an excessive inflammatory response that was beneficial in the presented case. $(7,8)$ Controlling pro-inflammatory response with hemoadsorption may have a positive impact on the endothelial glycocalyx and could lead to faster hemodynamic and metabolic stabilization resulting in preserved organ functions. The presented report is limited in one case and no conclusions can be made on specific airway management during combined therapy for heart and lung pathologies. This could be used as a material for an overview of possibilities for infective endocarditis patients with co-infections that results in additional support therapies.

\section{Conflict of interest: None}


Table 1. Chronology of hospitalization

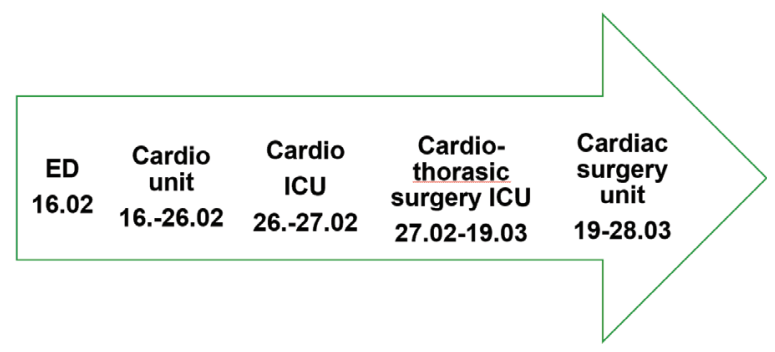

Table 2. Blood gas analysis during v-v ECMO

\begin{tabular}{|c|c|c|c|c|c|c|c|}
\hline & 27.02.2018 & 27.02 .2018 & 27.02.2018 & 28.02 .2018 & 01.03 .2018 & 02.03 .2018 & 03.03.2018 \\
\hline & NIV & NIV & $\mathrm{Sp}+\uparrow \mathrm{O}_{2} \mathrm{~m}$ & $\mathrm{Sp}+\uparrow \mathrm{O}_{2} \mathrm{~m}$ & $\mathrm{Sp}+\uparrow \mathrm{O}_{2} \mathrm{~m}$ & $\mathrm{Sp}+\uparrow \mathrm{O}_{2} \mathrm{~m}$ & $\mathrm{Sp}+\uparrow \mathrm{O}_{2} \mathrm{~m}$ \\
\hline $\mathrm{PaO}_{2}, \mathrm{mmHg}$ & 57 & 310 & $108-119$ & $106-164$ & $84-144$ & $94-113$ & $115-68$ \\
\hline $\mathrm{PaCO}_{2}, \mathrm{mmHg}$ & 30 & 29 & $27-33$ & $27-29$ & $23-29$ & $24-30$ & $21-29$ \\
\hline Lac, $\mathrm{mmol} / \mathrm{L}$ & 3,0 & 3,2 & $1,5-2,1$ & $1,1-1,4$ & $0,9-1,1$ & $1,0-2,0$ & $1,1-1,4$ \\
\hline$H t, \%$ & 31 & 25 & $24-25$ & $21-25$ & $23-25$ & $22-25$ & 28 \\
\hline$p H$ & 7,29 & 7,47 & $7,43-7,50$ & $7,50-7,53$ & $7,46-7,52$ & $7,44-7,47$ & $7,48-7,41$ \\
\hline$L / \min E C M O$ & - & 2.0 & 2,0 & 2,0 & 2,0 & 2,0 & 2,0 \\
\hline $\mathrm{FiO}_{2}$ & 1,0 & $\begin{array}{c}1,0 \\
\text { ECMO }\end{array}$ & $\begin{array}{c}1,0 \\
\text { ECMO }\end{array}$ & $\begin{array}{c}0,9 \\
\text { ECMO }\end{array}$ & $\begin{array}{c}0,8 \\
\text { ECMO }\end{array}$ & $\begin{array}{c}0,45-0,3 \\
\text { ECMO }\end{array}$ & $\begin{array}{c}0,3 \\
\text { ECMO }\end{array}$ \\
\hline$T,{ }^{\circ} \mathrm{C}$ & $37,5-38,0$ & $36,2-36,6$ & $35,2-36,8$ & $36,5-36,8$ & $36,5-36,9$ & $36,2-36,6$ & $37,0-37,5$ \\
\hline
\end{tabular}

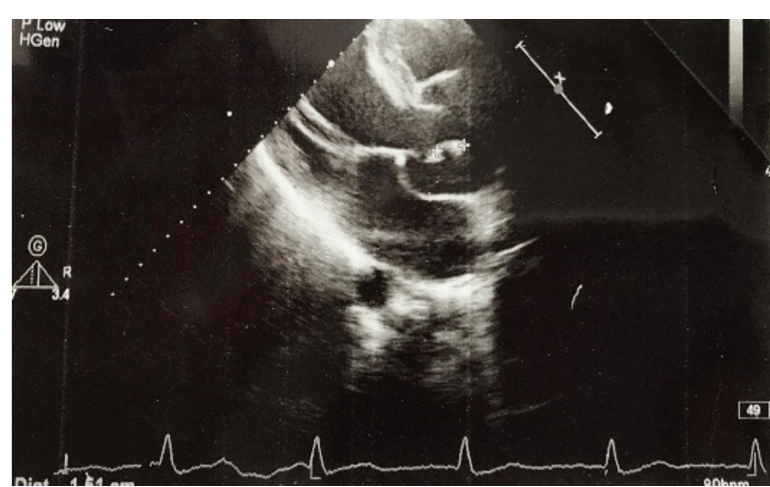

Fig. 1. EchoCG 19.02.2018 - additional formation on aorticnvalve Pauls Stradins Clinical university hospital

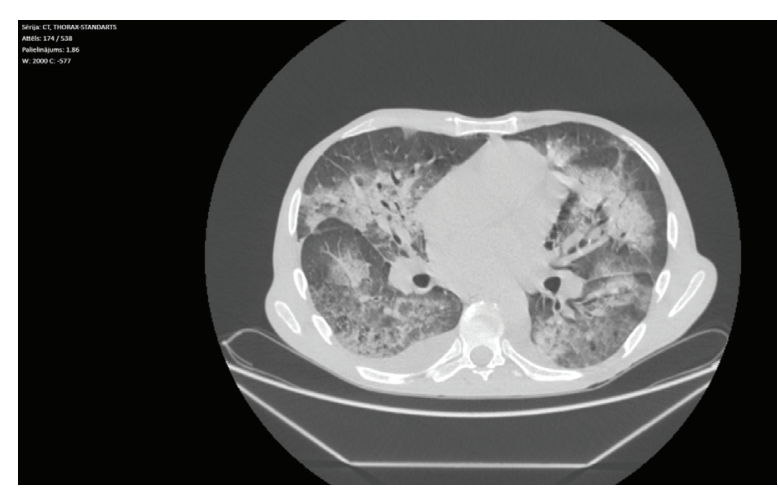




\section{REFERENCES}

1. The Third International Consensus Definitions for Sepsis and Septic Shock (Sepsis-3) I Critical Care Medicine I JAMA I JAMA Network [Internet]. [cited 2020 Apr 29]. Available from: https://jamanetwork. com/journals/jama/fullarticle/2492881

2. Rudd KE, Johnson SC, Agesa KM, Shackelford KA, Tsoi D, Kievlan DR, et al. Global, regional, and national sepsis incidence and mortality, 19902017: analysis for the Global Burden of Disease Study. The Lancet. 2020 Jan;395(10219):200-11.

3. Langer T, Santini A, Bottino N, Crotti S, Batchinsky AI, Pesenti A, et al. "Awake" extracorporeal membrane oxygenation (ECMO): pathophysiology, technical considerations, and clinical pioneering. Crit Care. 2016 30;20(1):150.

4. ELSO Guidelines for Adult Respiratory Failure 1_4.pdf [Internet]. [cited 2020 Apr 29]. Available from: https://www.elso.org/Portals/0/ELSO $\% 20$ Guidelines\%20For\%20Adult\%20Respiratory \%20 Failure\%201_4.pdf

5. Sukhal S, Sethi J, Ganesh M, Villablanca PA, Malhotra AK, Ramakrishna H. Extracorporeal membrane oxygenation in severe influenza infection with respiratory failure: A systematic review and meta-analysis. Ann Card Anaesth. 2017 Mar;20(1):14-21.
6. Koch CG, Khandwala F, Blackstone EH. Healthrelated quality of life after cardiac surgery. Semin Cardiothorac Vasc Anesth. 2008 Sep;12(3):20317.

7. Datzmann T, Träger K. Extracorporeal membrane oxygenation and cytokine adsorption. J Thorac Dis. 2018 Mar;10(Suppl 5):S653-60.

8. Hemoadsorption treatment of patients with acute infective endocarditis during surgery with cardiopulmonary bypass - a case series. PubMed - NCBI [Internet]. [cited 2020 Apr 29]. Available from: https://www.ncbi.nlm.nih.gov/ pubmed/28525670

\section{Address:}

Anna Klesmite

Paula Stradins Clinical university hospital

Pilsonu street 13

Riga, LV-1002, Latvia

E-mail: anna.klesmite@gmail.com 